\title{
Smart Environments: Technology to Support Healthcare
}

\author{
Dewar D. Finlay \\ University of Ulster, School of Computing and Mathematics, Faculty of Computing and Engineering, \\ Jordanstown, BT37 OQB Newtownabbey, Northern Ireland, UK
}

Ambient assistive living refers to the seamless integration of technology into a person's everyday environment in order to provide some form of assistance or support. Smart homes are one particular approach where specific emphasis is placed on the person's home environment. When extended beyond the home to the broader environments which the user may encounter on a daily basis the term "smart environment" is often used. Regardless of the terminology much effort is currently being expended in the development of these systems. In all applications the primary goal is to provide systems that offer some form of assistance or support. This can range from providing mechanisms that allow individuals to conduct every day tasks to systems that can monitor and advise on health status. This special issue of Technology and Health Care is made of contributions reporting recent technological developments in this research area. A total of eight papers are presented that cover topics from strategies for data collection to diary systems for weight management.

The issue begins with a paper by Szewcyzk et al. who have reported on a study that evaluates techniques that can be used to capture research data from smart environments. Specifically they have looked at how data sampled during research studies can be accurately labelled with the corresponding activity that is being monitored. The study found that, although potentially cumbersome for the subject, the most effective data collection method was to combine simulator data with a self report written diary of the subject being monitored. Also on the topic of smart environment data collection/generation Poland et al. report on a software tool that is being developed to allow computerised simulation of smart environments. This tool is being used to support work in the development of algorithms for optimal placement of sensors. One of the advantages of this approach is that it facilitates diverse configurations of real world environments to be simulated without the need for physical deployment.

Several of the papers describe work in the area of home monitoring. Skubic et al. report on their ongoing work in the development of systems for monitoring of elderly subjects in their own homes. They describe how data recorded from a range of sensors has the potential to highlight physical or cognitive decline. They also envisage the future roll-out of these systems to real homes away from the research setting. Fekki et al., again with specific emphasis on elderly subjects, present an overview of ongoing work in their laboratory. This work focuses on modelling daily activities of elderly subjects using home based sensors. They rely on low-level activity recognition algorithms the outcomes of which are fed to a larger classification framework. Kelly et al. have conducted research into location tracking 
systems for use in smart environments. They studied various radio signal parameters that are synonymous with current mobile devices, such as mobile telephones, in order to determine which of these signals could most effectively be used to determine a subject's position in a room. They show how the signals from such devices, along with computerised algorithms, can accurately determine a subject's location. The study highlights the trade-offs between device complexity and cost and detection functionality. Torres et al. have reported on the development of a Mediated Reality Location Aware system (MERLA). This system is designed to assist with personal navigation. In particular they describe the investigation of visual navigation tools. They conclude that the investigated tools assist with navigation in a simulated environment.

In the final two papers technical innovations are explored to provide assistance to specific cohorts. In the penultimate article Blanson Henkemans et al. describe the evaluation of a system that has been designed to help people manage their weight. The system is based around an online diary and a persuasive computer assistant. This assistant was in the form of an animated household pet dubbed the "iCat". The study found that the persuasive computer assistant had a positive motivational impact, as subjects were shown to be more compliant and comfortable with the online diary. Finally, Cunningham et al. present a review of how technology can be used to assist people who suffer from Parkinson's Disease. The review identifies how mobile devices and enhanced user interfaces could help persons with this condition.

In summary, the collection of articles presented in this issue cover a number of important challenges in ambient assistive living research. It is envisaged that these articles will be of interest to all readers of the journal and should consolidate the ongoing work in this area and help to fuel new research ideas. I would like to express my gratitude towards to the editorial board of Technology and Health Care for giving me the opportunity to act as guest editor for this special issue. I would also like to thank all the authors for their excellent contributions and thank all those who kindly provided comprehensive reviews for the submitted articles. 